\title{
Anti-inflammatory effect of lifestyle changes in the Finnish Diabetes Prevention Study
}

\author{
C. Herder • M. Peltonen • W. Koenig • K. Sütfels • \\ J. Lindström $\cdot$ S. Martin • P. Ilanne-Parikka • \\ J. G. Eriksson • S. Aunola • \\ S. Keinänen-Kiukaanniemi • T. T. Valle • M. Uusitupa • \\ H. Kolb • J. Tuomilehto • \\ for the Finnish Diabetes Prevention Study Group
}

Received: 22 August 2008 / Accepted: 18 November 2008 / Published online: 8 January 2009

(C) Springer-Verlag 2008

\begin{abstract}
Aims/hypothesis Subclinical inflammation confers an increased risk of type 2 diabetes, cardiovascular disease, neurodegenerative disorders and other age-related chronic diseases. Physical activity and diet can attenuate systemic immune activation, but it is not known which individual components of a comprehensive lifestyle intervention are most effective in targeting subclinical inflammation.
\end{abstract}

Electronic supplementary material The online version of this article (doi:10.1007/s00125-008-1243-1) contains supplementary material, which is available to authorised users.

C. Herder $\cdot$ K. Sütfels $\cdot$ S. Martin $\cdot$ H. Kolb

Institute for Clinical Diabetology, German Diabetes Centre,

Leibniz Centre at Heinrich Heine University Düsseldorf,

Düsseldorf, Germany

M. Peltonen $\cdot$ J. Lindström $\cdot$ J. G. Eriksson $\cdot$ T. T. Valle $\cdot$

J. Tuomilehto

Diabetes Unit,

Department of Health Promotion and Chronic Disease Prevention,

National Public Health Institute,

Helsinki, Finland

\section{W. Koenig $(\bowtie)$}

Department of Internal Medicine II-Cardiology,

University of Ulm Medical Centre,

Albert-Einstein-Allee 23,

89081 Ulm, Germany

e-mail: wolfgang.koenig@uniklinik-ulm.de

P. Ilanne-Parikka

Diabetes Centre of the Finnish Diabetes Association and the

Research Unit of Tampere University Hospital,

Tampere, Finland
Methods We used data from the baseline examination and the 1 year follow-up of a subsample of 406 of 522 participants of the Finnish Diabetes Prevention Study (DPS) to estimate the effect of individual components of lifestyle intervention on C-reactive protein (CRP) and IL-6 levels, which represent the best characterised proinflammatory risk factors for type 2 diabetes. Changes in metabolic markers, dietary patterns and exercise were analysed to

J. G. Eriksson

Department of General Practice and Primary Health Care,

University of Helsinki,

Helsinki, Finland

S. Aunola

Department of Health and Functional Capacity,

National Public Health Institute,

Turku, Finland

S. Keinänen-Kiukaanniemi

Oulu University Hospital and Health Centre,

Oulu Deaconess Institute and Institute of Health Sciences,

University of Oulu,

Oulu, Finland

M. Uusitupa

Department of Clinical Nutrition, University of Kuopio,

Kuopio, Finland

J. Tuomilehto

Department of Public Health, University of Helsinki,

Helsinki, Finland

J. Tuomilehto

South Ostrobothnia Central Hospital,

Seinäjoki, Finland 
determine which were most strongly associated with the anti-inflammatory effect of lifestyle changes.

Results Lifestyle intervention reduced circulating levels of CRP $(p<0.001)$ and IL-6 $(p=0.060)$. Increases in fibre intake and moderate to vigorous leisure time physical activity (LTPA), but not total LTPA, predicted decreases in CRP and/or IL-6 and remained associated even after adjustment for baseline BMI or changes in BMI during the first year of the study. Changes in carbohydrate or fat intake were either weakly or not linked to reductions in CRP and IL-6.

Conclusions/interpretation The present study assessed the individual effects of dietary and physical activity measures on low-grade inflammation in individuals at high cardiometabolic risk. Our results underline the importance of moderate to vigorous LTPA and a diet rich in natural fibre, and this should be emphasised in lifestyle recommendations.

\section{Trial registration: ClinicalTrials.gov NCT00518167}

Funding: The study was funded by the European Foundation for the Study of Diabetes, the German Federal Ministry of Health, the Ministry of Innovation, Science, Research and Technology of the State of North Rhine-Westphalia, the German Diabetes Foundation (Deutsche Diabetes-Stiftung), the Department of Internal Medicine II-Cardiology at the University of Ulm, the Academy of Finland, the Juho Vainio Foundation, the Finnish Ministry of Education, the Novo Nordisk Foundation, the Yrjö Jahnsson Foundation, the Finnish Diabetes Research Foundation and EVO funds from Tampere and Kuopio University Hospital.

Keywords Anti-inflammatory C C-reactive protein .

Diabetes · Fibre · Finnish Diabetes Prevention Study · IL-6 . Inflammation · Lifestyle intervention · Physical activity . Prevention

$\begin{array}{ll}\text { Abbreviations } \\ \text { CRP } & \text { C-reactive protein } \\ \text { DPP } & \text { Diabetes Prevention Program } \\ \text { DPS } & \text { Diabetes Prevention Study } \\ \text { IGT } & \text { Impaired glucose tolerance } \\ \text { KIHD } & \begin{array}{l}\text { Kuopio Ischaemic Heart Disease Risk Factor } \\ \end{array} \\ \text { Study } \\ \text { LTPA } & \text { Leisure time physical activity } \\ \text { MET } & \text { Metabolic equivalent }\end{array}$

\section{Introduction}

Type 2 diabetes has become one of the most important chronic public health problems worldwide. Major risk factors include dietary imbalance, physical inactivity and obesity, which are typical of a Westernised lifestyle [1,2].
The impact of dietary patterns and exercise is emphasised by lifestyle intervention studies, which have shown that disease onset can be delayed or even prevented in a substantial proportion of at-risk individuals [3-7].

Prospective studies have demonstrated that elevated circulating concentrations of several acute-phase proteins, cytokines, chemokines and soluble adhesion molecules precede the development of type 2 diabetes by many years [8-14]. Among these immune mediators, C-reactive protein (CRP) and IL-6 have been investigated for potential associations with incident type 2 diabetes in most prospective studies. In the Finnish Diabetes Prevention Study (DPS), CRP was associated with an increased risk of progression from impaired glucose tolerance (IGT) to type 2 diabetes in the absence of intensive lifestyle intervention [15].

Lifestyle intervention attenuates subclinical inflammation $[16,17]$, and it is reasonable to assume that the protection from type 2 diabetes afforded by lifestyle changes can, at least in part, be attributed to anti-inflammatory effects. However, it is not clear which components of comprehensive lifestyle interventions are most closely related to attenuation of low-grade inflammation. Dietary patterns, physical activity and weight loss have all been reported to be associated with reduced circulating levels of acute phase proteins and cytokines [16, 18, 19], but have not been analysed in parallel for their relative contribution and relevance to reductions in proinflammatory markers.

In addition to type 2 diabetes, subclinical inflammation is also a risk factor for cardiovascular disease, neurodegenerative disorders, depression and some cancers [20-24], which means that the identification of the mechanisms that modulate and attenuate systemic immune activation has widespread implications.

Since participants in the Finnish DPS underwent extensive phenotyping, including anthropometric, metabolic and lifestyle factors at baseline and at follow-up visits [25-27], data from this study allows us to assess the effect of lifestyle changes on proinflammatory mediators in more detail compared with data from most other studies. Therefore, using data from the Finnish DPS, focusing specifically on CRP and IL-6, we investigated (1) whether lifestyle intervention affected these markers of subclinical inflammation, (2) whether changes in circulating levels of CRP and IL-6 were associated with changes in anthropometric and metabolic measures, and (3) which changes in dietary intake, exercise or weight were most strongly associated with the anti-inflammatory effect of the lifestyle intervention.

\section{Methods}

Participants The Finnish DPS is a multicentre, randomised, controlled trial that was designed to investigate whether a 
lifestyle intervention aimed at increasing physical activity, improving diet and decreasing body weight reduces the risk of developing type 2 diabetes in high-risk individuals. The study design has been described in detail elsewhere [25, 27]. Briefly, the study population consisted of 522 men and women aged 40-65 years who were overweight or obese $\left(\mathrm{BMI} \geq 25 \mathrm{~kg} / \mathrm{m}^{2}\right)$ and had IGT ( $2 \mathrm{~h}$ OGTT plasma glucose $7.8-11.0 \mathrm{mmol} / \mathrm{l}[140-200 \mathrm{mg} / \mathrm{dl}]$ and fasting plasma glucose $<7.8 \mathrm{mmol} / 1[<140 \mathrm{mg} / \mathrm{dl}]$ at baseline). The study protocol was approved by the ethics committee of the National Public Health Institute in Helsinki, Finland, and all study participants gave written informed consent.

The present study is based on a subsample of 406 participants. Sixteen of the 522 participants did not attend the 1 year follow-up appointment and can be considered as dropouts for this study. A further 100 participants were excluded because of missing baseline or 1 year serum samples.

Intervention Study participants were randomly assigned to either the intervention group $(n=265)$ or the control group $(n=257)[4,27]$. Participants in the intervention group were given detailed advice on how to achieve the goals of the intervention, which were: (1) moderate to vigorous exercise for $\geq 30 \mathrm{~min} /$ day, (2) a reduction in intake of fat to $<30 \%$ of total energy intake, (3) a reduction in intake of saturated fat to $<10 \%$ of total energy intake, (4) an increase in fibre intake to $\geq 15 \mathrm{~g}$ per $4,186 \mathrm{~kJ}(1,000 \mathrm{kcal})$, and (5) a reduction in body weight of $\geq 5 \%$. They were individually guided to increase their overall level of physical activity. This guidance was given by the nutritionist during the dietary counselling sessions and highlighted by the study physicians at the annual visits. Endurance exercise was recommended to increase aerobic capacity and cardiorespiratory fitness. Supervised, progressive, individually tailored, circuit-type, moderate intensity resistance training sessions to improve the functional capacity and strength of the large muscle groups of the upper and lower body were also offered, free of charge, beginning 4-6 months after randomisation. At baseline, the control group was given general information about lifestyle and diabetes risk. This was done either individually or in a group session, and printed material was delivered.

Anthropometric, clinical and lifestyle assessments Measurements of height, weight, waist circumference and blood pressure have been described in detail previously [25]. At baseline and at each annual visit, all participants underwent a $2 \mathrm{~h}$ OGTT and completed the validated Kuopio Ischaemic Heart Disease Risk Factor Study (KIHD) 12 month Leisure Time Physical Activity (LTPA) Questionnaire [28, 29] and a 3 day food diary [30]. The 12 month LTPA questionnaire is a detailed quantitative questionnaire that allows the estimation of frequency, duration and intensity of the participants' most common lifestyle and structured LTPA as recalled over the previous 12 months. Amounts of total LTPA and moderate to vigorous LTPA were calculated and expressed as hours per week. Moderate to vigorous LTPA was defined as $\geq 3.5$ metabolic equivalents (METs), where 1 MET represents metabolic expenditure at rest, corresponding to an oxygen uptake of $3.5 \mathrm{ml} \mathrm{O}_{2} / \mathrm{kg}$ [28]. The 3 day food diary was completed using a picture booklet showing the portion sizes of typical foods. Nutrient intakes were calculated using a dietary analysis program developed in the National Public Health Institute (Helsinki, Finland) [31]. Importantly, dietary data collection was performed by identical methods in both groups, to avoid a bias in the reporting of nutritional habits.

Blood sampling and laboratory measurements Blood samples, taken from the antecubital vein with the participant in a sitting position, were allowed to clot at room temperature for 30-60 min. After centrifugation at $8,000-11,000 \times g$ for $15 \mathrm{~min}$ at room temperature $\left(20-25^{\circ} \mathrm{C}\right)$, the serum layer was removed and stored at $-70^{\circ} \mathrm{C}$ for future analyses although for logistic reasons, storage at $-20^{\circ} \mathrm{C}$ was allowed for a maximum of 3 months. Serum concentrations of CRP were assessed by an immunonephelometric assay (Dade Behring, Marburg, Germany) [15]. Serum IL-6 concentrations were determined by ELISA using recombinant IL6 and an antibody pair from Sanquin (Amsterdam, the Netherlands) [15]. Concentrations of plasma glucose, serum insulin, total cholesterol and LDL-cholesterol were assessed by means of standard methods in the central laboratory in Helsinki as described in detail previously [4, 25]. Insulin resistance measured by homeostasis model assessment was calculated as follows: HOMA-IR=fasting glucose $(\mathrm{mmol} / \mathrm{l}) \times$ fasting insulin $(\mathrm{pmol} / \mathrm{l}) / 135$.

Statistical analysis Data at study baseline and 1 year are presented as means $\pm \mathrm{SD}$, except for CRP and IL-6 (given as median and interquartile range), and were compared with paired Student's $t$ tests (two-tailed). Changes from baseline at 1 year are shown as means $(95 \% \mathrm{CI})$. Differences in changes from baseline at 1 year between control and intervention groups were compared using unpaired Student's $t$ tests (two-tailed). Correlations between changes in immunological markers (concentration at 1 year-concentration at baseline) and corresponding changes in other variables were assessed by using Spearman's coefficients of correlation. Nominal $p$ values are shown without adjustment for multiple comparisons. Partial Spearman correlation and its $p$ value were used to assess the association between changes in two variables when adjusting for other covariates. All correlation analyses were performed in both control and intervention groups combined. Associations with $p$ values of $<0.05$ were considered statistically 
significant. Statistical analyses were performed using Stata Statistical Software Package, Release 8.0 (StataCorp, College Station, TX, USA).

\section{Results}

Lifestyle intervention attenuates systemic low-grade inflammation The present study comprised 406 participants for whom data and serum samples from baseline and 1 year were available (control group: $n=199$ [67 men, 132 women], intervention group: $n=207$ [74 men, 133 women]). A dropout analysis showed that the 116 individuals who were not included in this study did not differ from the 406 participants in age, BMI, waist circumference, fasting glucose, fasting insulin, HOMA-IR, CRP or IL-6 levels at baseline (data not shown).

There was a significant decrease in serum concentrations of CRP during the first study year in the intervention group (mean change: $-1.24 \mathrm{mg} / 1, p<0.001$; control group $-0.38 \mathrm{mg} / 1, p=0.12 ; p=0.053$ for the difference between the groups) (Fig. 1a,b). Likewise IL-6 concentrations tended to decrease in the intervention group $(-0.40 \mathrm{pg} / \mathrm{ml}, p=0.060)$ but not in the control group $(+0.22 \mathrm{pg} / \mathrm{ml}, p=0.27)$ (Fig. $1 \mathrm{c}, \mathrm{d})$, and the betweengroup difference was statistically significant $(p=0.033)$.
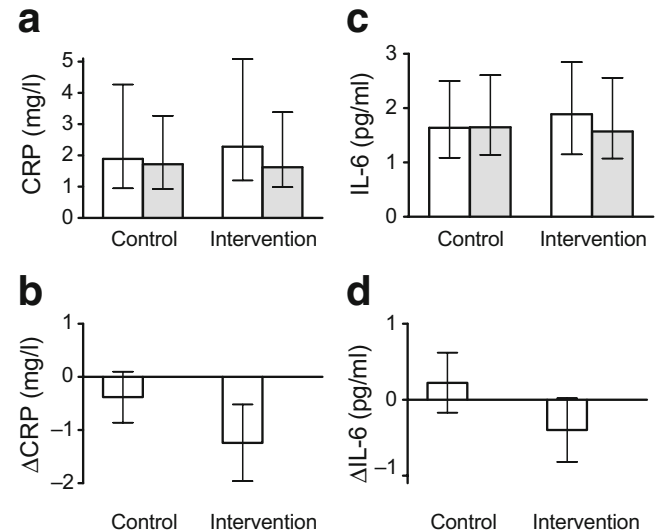

Fig. 1 Changes at 1 year in serum concentrations of CRP and IL-6 in the control group $(n=199)$ and the intervention group $(n=207)$. a Median and interquartile range of CRP levels at baseline (white bars) and after 1 year (grey bars); $p=0.0003$ for levels at 1 year vs baseline for the intervention group. b Mean and $95 \%$ CIs of CRP changes between study baseline and 1 year; $p=0.060$ for intervention vs control. c Median and interquartile range of IL-6 levels at baseline (white bars) and after 1 year (grey bars); $p=0.053$ for levels at 1 year vs baseline for the intervention group. d Mean and 95\% CIs of IL-6 changes between study baseline and 1 year $1 ; p=0.033$ for intervention vs control
There was a significant correlation between baseline levels of CRP and IL-6 $(r=0.358, p<0.001)$, and changes in CRP and IL-6 were also significantly correlated $(r=0.196$, $p<0.001)$. IL-6 levels explained $12.8 \%$ of the variance in baseline CRP levels and vice versa. Adjustment for age and sex or for age, sex and BMI reduced the explained variance to $11.9 \%$ and $7.3 \%$, respectively.

Changes in CRP and IL-6 levels and anthropometric and metabolic variables at 1 year Participants in the intervention group showed more pronounced reductions in measures of obesity, fasting and OGTT $2 \mathrm{~h}$ glucose, insulin resistance and blood pressure (Table 1). Decreases in CRP levels were associated with weight loss, reductions in waist circumference, systolic and diastolic blood pressure and fasting glucose levels, and improvements in HOMA-IR (Electronic Supplementary Material [ESM] Table 1). Adjustment for age, sex, baseline BMI and treatment group attenuated the correlation between changes in CRP and HOMA-IR, whereas the other associations remained relatively stable. Since CRP levels were strongly associated with BMI, we determined variables that changed with decreasing CRP levels independently of any reductions in weight. For this purpose, we additionally adjusted for changes in BMI and found that only changes in diastolic blood pressure occurred in parallel with alterations in CRP levels independently of weight loss.

The pattern of associations between changes in IL- 6 and changes in anthropometric and metabolic variables was similar to that for CRP, but the associations were generally less pronounced (ESM Table 1). Decreases in IL-6 were significantly associated with decreases in weight, waist circumference, fasting insulin and diastolic blood pressure, but were not associated with changes in fasting glucose, HOMA-IR or systolic blood pressure. Adjustment for age, sex, baseline BMI and treatment group reduced the strength of the associations for these variables, and they were no longer significant after also adjusting for changes in weight.

Changes in CRP and IL-6 levels and lifestyle variables at 1 year Significant changes in lifestyle variables occurred over the first year of the trial to permit the investigation of an association with changes in low-grade inflammation. Participation in the control group led to dietary changes: a lower energy intake and modest changes in food composition (Table 2). The dietary changes were more pronounced in the intervention group, who also showed a significant increase in the amount of moderate to vigorous LTPA (Table 2). The correlation was high $(r=0.77)$ between changes in fat and saturated fat intake, moderate $(r=-0.22)$ between changes in fat and fibre, and non-significant between changes in physical activity and fat intake. 


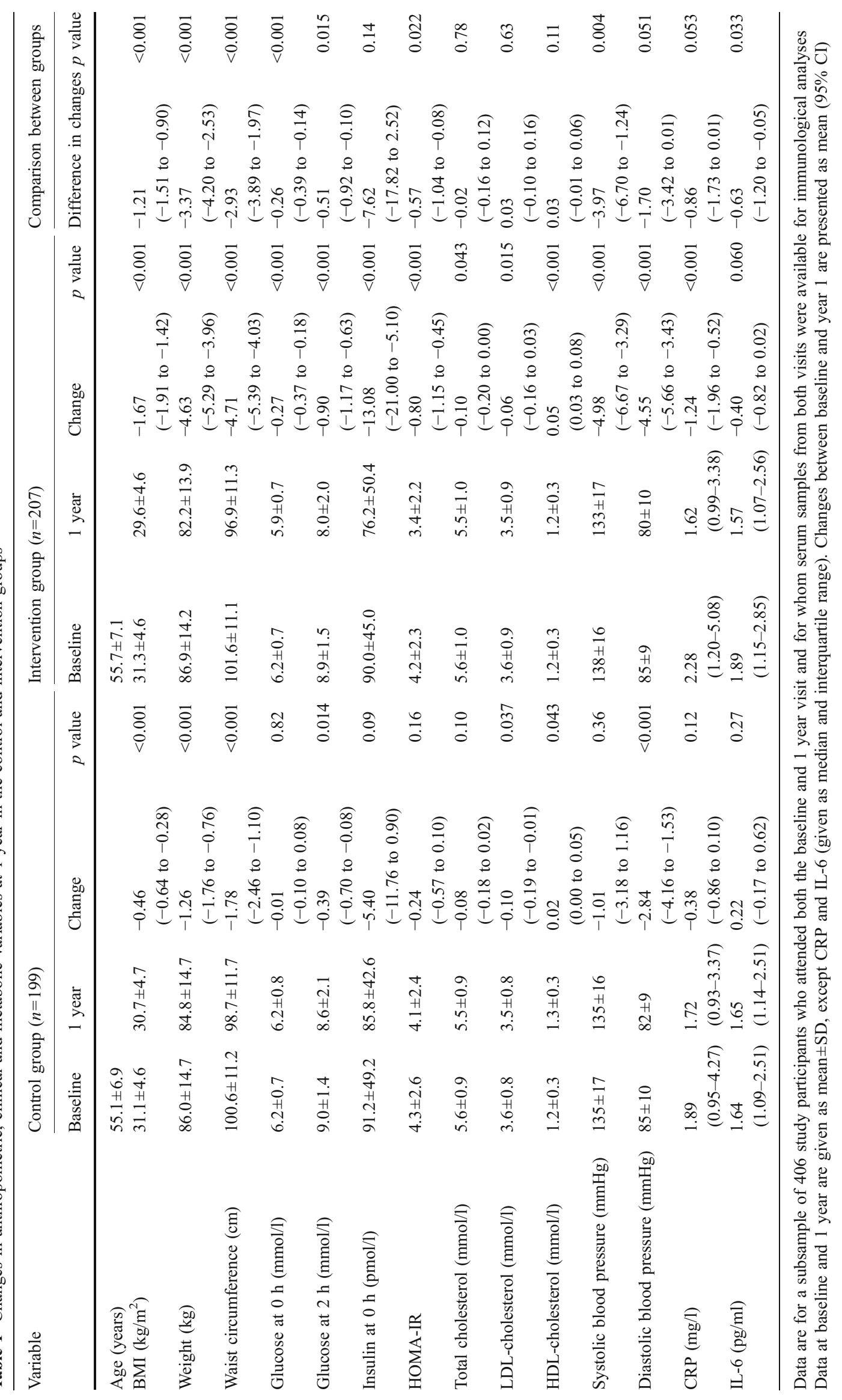




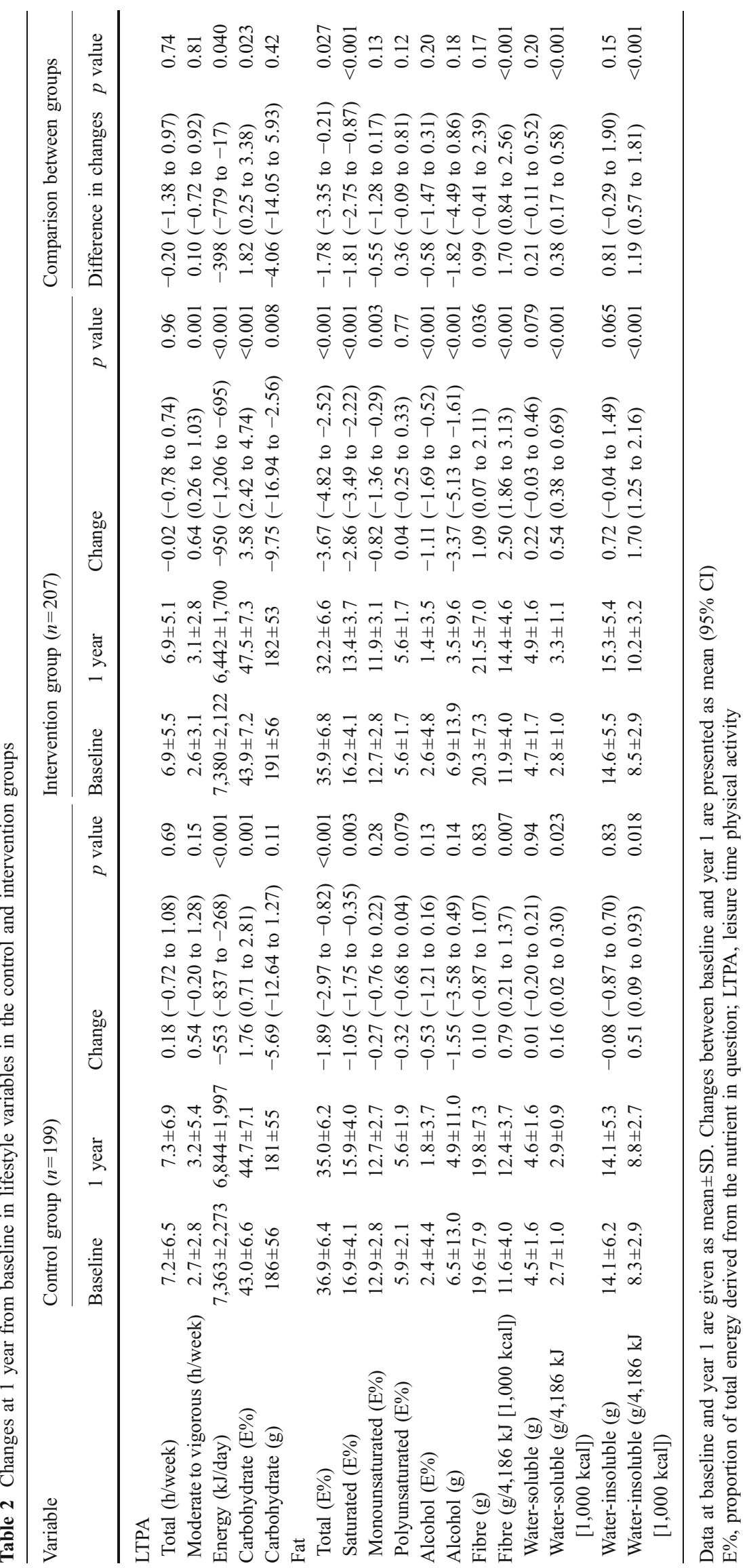


Table 3 Correlation of changes at 1 year from baseline in concentrations of systemic immune mediators with changes in LTPA and dietary components over the same period

\begin{tabular}{|c|c|c|c|c|c|c|c|c|c|c|c|c|}
\hline \multirow[t]{3}{*}{ Variable } & \multicolumn{6}{|c|}{ Changes in CRP } & \multicolumn{6}{|c|}{ Changes in IL-6 } \\
\hline & \multicolumn{2}{|c|}{ Unadjusted } & \multicolumn{2}{|c|}{$\begin{array}{l}\text { Adjusted for } \\
\text { age, sex, group } \\
\text { and baseline } \\
\text { BMI }\end{array}$} & \multicolumn{2}{|c|}{$\begin{array}{l}\text { Adjusted for } \\
\text { age, sex, group, } \\
\text { baseline BMI } \\
\text { and } \Delta \mathrm{BMI}\end{array}$} & \multicolumn{2}{|c|}{ Unadjusted } & \multicolumn{2}{|c|}{$\begin{array}{l}\text { Adjusted for } \\
\text { age, sex, group } \\
\text { and baseline } \\
\text { BMI }\end{array}$} & \multicolumn{2}{|c|}{$\begin{array}{l}\text { Adjusted for } \\
\text { age, sex, group } \\
\text { baseline BMI } \\
\text { and } \Delta \mathrm{BMI}\end{array}$} \\
\hline & $r$ & $\begin{array}{l}p \\
\text { value }\end{array}$ & $r$ & $\begin{array}{l}p \\
\text { value }\end{array}$ & $r$ & $\begin{array}{l}p \\
\text { value }\end{array}$ & $r$ & $\begin{array}{l}p \\
\text { value }\end{array}$ & $r$ & $\begin{array}{l}p \\
\text { value }\end{array}$ & $r$ & $\begin{array}{l}p \\
\text { value }\end{array}$ \\
\hline \multicolumn{13}{|l|}{ LTPA } \\
\hline Total (h/week) & -0.048 & 0.35 & -0.032 & 0.54 & 0.017 & 0.74 & -0.042 & 0.41 & -0.047 & 0.36 & -0.022 & 0.67 \\
\hline Moderate to vigorous (h/week) & -0.130 & 0.011 & -0.124 & 0.015 & -0.092 & 0.074 & -0.164 & 0.001 & -0.152 & 0.003 & -0.135 & 0.008 \\
\hline Energy (kJ/day) & 0.122 & 0.015 & 0.098 & 0.051 & 0.070 & 0.16 & -0.034 & 0.50 & -0.047 & 0.35 & -0.061 & 0.22 \\
\hline Carbohydrates (E\%) & -0.075 & 0.14 & -0.052 & 0.31 & -0.033 & 0.52 & -0.022 & 0.67 & -0.003 & 0.96 & 0.006 & 0.90 \\
\hline Carbohydrates (g) & 0.092 & 0.068 & 0.072 & 0.15 & 0.055 & 0.28 & -0.051 & 0.31 & -0.058 & 0.25 & -0.067 & 0.19 \\
\hline \multicolumn{13}{|l|}{ Fat } \\
\hline Total $(\mathrm{E} \%)$ & 0.074 & 0.004 & 0.039 & 0.44 & -0.003 & 0.96 & 0.029 & 0.56 & 0.010 & 0.85 & -0.007 & 0.88 \\
\hline Saturated $(\mathrm{E} \%)$ & 0.145 & 0.004 & 0.106 & 0.035 & 0.078 & 0.12 & 0.039 & 0.44 & 0.015 & 0.77 & 0.001 & 0.99 \\
\hline Monounsaturated (E\%) & 0.076 & 0.13 & 0.048 & 0.34 & 0.015 & 0.77 & 0.060 & 0.23 & 0.044 & 0.39 & 0.029 & 0.57 \\
\hline Polyunsaturated (E\%) & -0.065 & 0.20 & -0.039 & 0.44 & -0.056 & 0.26 & -0.014 & 0.78 & 0.005 & 0.93 & -0.003 & 0.96 \\
\hline Alcohol (E\%) & -0.065 & 0.20 & -0.054 & 0.29 & -0.075 & 0.14 & -0.059 & 0.24 & -0.061 & 0.23 & -0.070 & 0.17 \\
\hline Alcohol (g) & -0.041 & 0.42 & -0.028 & 0.57 & -0.050 & 0.32 & -0.049 & 0.33 & -0.051 & 0.32 & -0.060 & 0.23 \\
\hline Fibre $(\mathrm{g})$ & -0.093 & 0.064 & -0.090 & 0.073 & -0.080 & 0.11 & -0.143 & 0.004 & -0.137 & 0.006 & -0.132 & 0.008 \\
\hline Fibre (g/4,186 kJ [1,000 kcal]) & -0.184 & $<0.001$ & -0.154 & 0.002 & -0.122 & 0.015 & -0.089 & 0.075 & -0.068 & 0.18 & -0.052 & 0.31 \\
\hline Water-soluble (g) & -0.059 & 0.24 & -0.062 & 0.22 & -0.051 & 0.31 & -0.136 & 0.006 & -0.132 & 0.010 & -0.126 & 0.012 \\
\hline Water-soluble (g/4,186 kJ [1,000 kcal]) & -0.147 & 0.003 & -0.122 & 0.015 & -0.089 & 0.082 & -0.108 & 0.031 & -0.091 & 0.071 & -0.075 & 0.14 \\
\hline Water-insoluble (g) & -0.100 & 0.045 & -0.102 & 0.042 & -0.093 & 0.065 & -0.134 & 0.007 & -0.129 & 0.010 & -0.125 & 0.013 \\
\hline $\begin{array}{l}\text { Water-insoluble }(\mathrm{g} / 4,186 \mathrm{~kJ} \\
\quad[1,000 \mathrm{kcal}])\end{array}$ & -0.192 & $<0.001$ & -0.168 & 0.001 & -0.139 & 0.006 & -0.088 & 0.078 & -0.069 & 0.17 & -0.054 & 0.29 \\
\hline
\end{tabular}

Data are for intervention and control groups combined, $n=406$

Correlations are given as (partial) Spearman correlation coefficients, $r$

$\mathrm{E} \%$, proportion of total energy derived from the nutrient in question

The decrease in systemic CRP concentrations over the first year was associated both with an increased amount of moderate to vigorous LTPA and with changes in dietary pattern (i.e. decreased total energy intake, decreased proportions of total energy derived from fat and saturated fat and increased amount of fibre) (Table 3). The association with fibre was independent of body weight (Table 3).

Changes in IL-6 were also found to be associated with an increased amount of moderate-to-vigorous LTPA, but among the dietary components, only an increased fibre intake (total, water-soluble and water-insoluble) was found to be associated (Table 3). All associations were independent of weight loss.

Sensitivity analysis To exclude an influence of acute infections or other proinflammatory conditions at baseline or the 1 year follow-up visit on our results we excluded all study participants with CRP levels of $>10 \mathrm{mg} / \mathrm{l}$ at one or both examinations $(n=26)$ and repeated the analyses shown in Table 3 and ESM Table 1. Associations between immune marker changes and changes in other variables either remained stable or were only slightly attenuated, and all associations with a $p$ value of $\leq 0.031$ in the original analysis remained significant in this sensitivity analysis (data not shown).

\section{Discussion}

This study represents the first comparison of the effect of increased LTPA and improved diet quality on low-grade inflammation in individuals at high risk of type 2 diabetes. Since subclinical inflammation increases the risk of cardiovascular disease, disability, neurodegenerative disorders and cancers [20-24], it is important to identify efficient ways to modulate low-grade immune activation. Lifestylebased interventions are one option as they have been proved to exert anti-inflammatory effects in many studies $[17,32]$. The decrease in CRP and IL-6 levels in response to lifestyle intervention in the Finnish DPS reported here is 
similar to the effects observed in other studies. In the Diabetes Prevention Program (DPP), lifestyle intervention achieved median reductions in CRP levels of 33\% and 29\% between baseline and 1 year in men and women, respectively [16], compared with a median relative reduction in CRP of $27 \%$ in the intervention group of the Finnish DPS. When estimating CRP changes per unit of weight loss, a systematic review based on 33 studies showed that, for each $\mathrm{kg}$ of weight loss, mean CRP levels declined by $0.13 \mathrm{mg} / 1$ [17], whereas a longitudinal study from the UK reported a linear association between CRP levels and weight gain over 9 years (CRP $+0.09 \mathrm{mg} \mathrm{l}^{-1} \mathrm{~kg}^{-1}$ ) [33]. We report mean CRP reductions and weight loss of $1.24 \mathrm{mg} / \mathrm{l}$ and $4.63 \mathrm{~kg}$, respectively, in the intervention group, which resulted in a CRP change of $-0.27 \mathrm{mg}^{-1}[\mathrm{~kg}$ weight loss $]^{-1}$. Bearing in mind that weight loss is not the only determinant of changes in CRP, the strength of the association between early changes in CRP and weight loss appears to be similar or even greater in the Finnish DPS than in the previously conducted intervention studies.

In the Finnish DPS, almost all investigated anthropometric and metabolic markers showed favourable changes in the intervention group. However, only some of these changes were associated with changes in CRP or IL-6 during the first study year. We found that the strongest associations of reductions in CRP and IL-6 were with improvements in indexes of obesity (BMI, weight and waist circumference; all $p<0.01$ ).

Significant associations between changes in CRP and/or IL-6 with markers of glucose metabolism were present in the unadjusted analysis, but were attenuated when baseline BMI and changes in BMI during the first study year were included in the model. In addition, we found a robust association of CRP changes (less so for changes in IL-6) with changes in diastolic blood pressure, but not with changes in lipid levels.

The lack of associations between changes in immune mediators and changes in lipid levels may be due to the fact that the effect of the intervention on lipids was rather small in the Finnish DPS. As shown in Table 1, there were no significant differences in changes in total cholesterol, HDLcholesterol or LDL-cholesterol when the control and intervention groups were compared. Therefore, a lack of variation of lipid changes could have made it more difficult to find an association in the present study than in a study that was primarily designed to alter the lipid status of the participants.

These data extend findings from the DPP, in which associations of changes in CRP with changes in BMI and waist circumference were strong, whereas associations with changes in glucose, insulin and HOMA-IR were weaker (partial Spearman coefficients of between 0.09 and 0.11, adjusted for age, sex, race and treatment group) [16]. Changes in blood pressure or lipids were not included in the
DPP analyses. As lifestyle intervention in both the DPS and DPP resulted in considerable protection from the development of type 2 diabetes, it is not quite clear why changes in subclinical inflammation and insulin resistance were not more strongly related in the two studies. One explanation could be that intraindividual variation in immunological variables may be higher than the variation in anthropometric and metabolic markers, such that serial measurements of immune mediators at baseline and during follow-up would allow a more precise assessment of the effect of any intervention on immune status. Alternatively, it may be that the inflammatory markers studied are not those most closely linked to the disease process, or that the influence of subclinical inflammation on insulin resistance is smaller than that estimated based on numerous previous studies (reviewed in [20]).

Like the anthropometric and metabolic markers analysed here, most measures of diet and LTPA also showed favourable changes in the intervention group. Again, only some of these changes predicted reductions in CRP and/or IL-6. The lifestyle changes robustly associated with reductions in CRP and IL-6 levels were increases in time spent partaking in moderate to vigorous LTPA and increases in fibre intake. Importantly, most of these associations remained significant even after adjustment for baseline BMI and changes in BMI. Thus, our data expand current knowledge on the relationship between lifestyle and inflammation, which up to now has mainly been based on correlation analyses in cross-sectional studies.

An important major finding was the strong association between changes in subclinical inflammation and changes in time spent doing moderate to vigorous LTPA, although changes in low-intensity as well as in total LTPA were at least as good as predictors of diabetes incidence as changes in time spent doing moderate to vigorous LTPA [28]. This indicates that a higher intensity of physical activity may be necessary to reduce systemic low-grade inflammation than is needed to prevent or delay the incidence of type 2 diabetes in individuals at high risk of the disease, and points towards inflammation-independent effects of lowintensity LTPA. It is also important to note that the associations between increases in LTPA and reductions in CRP and IL-6 were only slightly affected when adjusted for weight loss $(p=0.074$ and $p=0.008$, respectively). This suggests an obesity-independent component of the relationship between moderate to vigorous LTPA and subclinical inflammation, which seems reasonable as it is known that obesity and physical activity are independent risk factors for type 2 diabetes, cardiovascular disease and mortality [34, 35]. Moreover, our findings emphasise that the association between physical activity and subclinical inflammation is rather complex, as regular exercise seems to lower circulating levels of cytokines such as IL-6, whereas medium- to high-intensity exercise induces the 
acute release of IL-6 from muscle tissue [36]. According to our current understanding of the role of IL- 6 , although it exerts beneficial effects locally (i.e. in muscle), elevated expression of the gene encoding IL- 6 in adipose tissue and leucocytes is a hallmark of obesity, the metabolic syndrome, type 2 diabetes and cardiovascular disease risk [10, 21, 37-40].

Our study extends previous reports indicating that higher fibre intake is associated with reduced subclinical inflammation in cross-sectional [41, 42], prospective [43] and intervention studies [44, 45], as it identifies changes in both water-soluble and water-insoluble fibre intake as dominant contributors to the reduction in systemic inflammation in a complex lifestyle intervention setting. In particular, the associations between changes in IL- 6 and changes in fibre intake were independent of weight loss and therefore point towards an obesity-independent beneficial effect of fibre on health. This is supported by previously published data demonstrating that high fibre intakes predict decreased diabetes risk independently of body weight change in the Finnish DPS [46]. It has been hypothesised that dietary fibre decreases lipid oxidation and thereby attenuates subclinical inflammation [47]. In addition, a diet rich in natural fibre may contain components other than fibre per se that could contribute to the health benefit of fibre-rich diets.

An interesting finding from our study was that changes in CRP and IL-6 showed different associations with lifestyle changes. IL-6 is the main inductor of CRP production in the liver [48], and so similar effects on these two immune mediators would be expected. However, a correlation between CRP and IL-6 levels at baseline of $r=0.358$ means that only $12.8 \%$ of variance in CRP levels was explained by IL-6 and vice versa, and adjustment for age and sex or for age, sex and BMI reduced the explained variance even further. This indicates that measurements of CRP and IL- 6 do not provide identical information and points towards substantial differences in the regulation of the two immune mediators.

This study has certain limitations that should be mentioned. Our sample consisted of individuals with IGT, i.e. at high risk of cardiometabolic disease, and is therefore not representative of the general population. In addition, our sample size was not sufficiently large to allow meaningful stratified analyses to be conducted or to test for effect modifications by age, sex, obesity or other variables. Evaluation of diet and LTPA was based on memory and/or self-reports; therefore, more objective measurements may have resulted in more pronounced associations with low-grade inflammation. Food diaries are considered a reliable method for dietary studies, but under- or misreporting can occur. Mean changes in some lifestyle components, such as intake of polyunsaturated fat, were small; consequently, it is possible that beneficial effects on inflammation were not seen that would have been apparent in an intervention trial that focused on single dietary components. However, the aim of this study was to compare the anti-inflammatory potential of different lifestyle factors and their components in the same study, which means that this study limitation was unavoidable.

In conclusion, our analysis of the anti-inflammatory effects of the intensive lifestyle intervention in the Finnish DPS revealed that changes in moderate to vigorous LTPA and changes in fibre intake were the two lifestyle variables most strongly associated with reductions in CRP and IL-6 in individuals at high cardiometabolic risk. Since subclinical inflammation confers an elevated risk of many agerelated and chronic diseases in addition to type 2 diabetes and cardiovascular disease, the importance of the intensity of LTPA and of a diet rich in natural fibre should be emphasised in lifestyle recommendations.

Acknowledgements Work was supported by the European Foundation for the Study of Diabetes, the German Federal Ministry of Health, the Ministry of Innovation, Science, Research and Technology of the State of North Rhine-Westphalia, the German Diabetes Foundation (Deutsche Diabetes-Stiftung), the Department of Internal Medicine IICardiology at the University of Ulm, the Academy of Finland (grants $8473 / 2298,40758 / 5767,38387 / 54175,46558$ ), the Juho Vainio Foundation, the Finnish Ministry of Education, the Novo Nordisk Foundation, the Yrjö Jahnsson Foundation, the Finnish Diabetes Research Foundation and EVO funds from Tampere and Kuopio University Hospital. We thank W. Mohné and G. Trischler for expert technical assistance. We appreciate the voluntary contribution of all study participants.

Duality of interest The authors declare that there is no duality of interest associated with this manuscript.

\section{References}

1. American Diabetes Association (2008) Nutrition recommendations and interventions for diabetes: a position statement of the American Diabetes Association. Diabetes Care 31:S61-S78

2. Kempf K, Rathmann W, Herder C (2008) Impaired glucose regulation and type 2 diabetes in children and adolescents. Diabetes Metab Res Rev 24:427-437

3. Pan XR, Li GW, Hu YH et al (1997) Effects of diet and exercise in preventing NIDDM in people with impaired glucose tolerance. The Da Qing IGT and Diabetes Study. Diabetes Care 20:537-544

4. Tuomilehto J, Lindström J, Eriksson JG et al (2001) Prevention of type 2 diabetes mellitus by changes in lifestyle among subjects with impaired glucose tolerance. N Engl J Med 344:1343-1350

5. Knowler WC, Barrett-Connor E, Fowler SE et al (2002) Reduction in the incidence of type 2 diabetes with lifestyle intervention or metformin. N Engl J Med 346:393-403

6. Ramachandran A, Snehalatha C, Mary S et al (2006) The Indian Diabetes Prevention Programme shows that lifestyle modification and metformin prevent type 2 diabetes in Asian Indian subjects with impaired glucose tolerance (IDPP-1). Diabetologia 49:289-297

7. Lindström J, Ilanne-Parikka P, Peltonen M, Aunola S, Eriksson JG (2006) Sustained reduction in the incidence of type 2 diabetes by lifestyle intervention. Follow-up of the Finnish Diabetes Prevention Study. Lancet 368:1673-1679 
8. Schmidt MI, Duncan BB, Sharrett AR et al (1999) Markers of inflammation and prediction of diabetes mellitus in adults (Atherosclerosis Risk in Communities study): a cohort study. Lancet 353:1649-1652

9. Barzilay JI, Abraham L, Heckbert SR et al (2001) The relation of markers of inflammation to the development of glucose disorders in the elderly: the Cardiovascular Health Study. Diabetes 50:2384-2389

10. Pradhan AD, Manson JE, Rifai N, Buring JE, Ridker PM (2001) C-reactive protein, interleukin 6, and risk of developing type 2 diabetes mellitus. JAMA 286:327-334

11. Meigs JB, Hu FB, Rifai N, Manson JE (2004) Biomarkers of endothelial dysfunction and risk of type 2 diabetes mellitus. JAMA 291:1978-1986

12. Thorand B, Kolb H, Baumert J et al (2005) Elevated levels of interleukin-18 predict the development of type 2 diabetes: results from the MONICA/KORA Augsburg Study, 1984-2002. Diabetes 54:2932-2938

13. Herder C, Baumert J, Thorand B et al (2006) Chemokines as risk factors for type 2 diabetes: results from the MONICA/KORA Augsburg Study, 1984-2002. Diabetologia 49:921-929

14. Herder C, Klopp N, Baumert J et al (2008) Effect of macrophage migration inhibitory factor (MIF) gene variants and MIF serum concentrations on the risk of type 2 diabetes: results from the MONICA/KORA Augsburg Case-Cohort Study, 1984-2002. Diabetologia 51:276-284

15. Herder C, Peltonen M, Koenig W et al (2006) Systemic immune mediators and lifestyle changes in the prevention of type 2 diabetes: results from the Finnish Diabetes Prevention Study. Diabetes 55:2340-2346

16. Diabetes Prevention Program Research Group (2005) Intensive lifestyle intervention or metformin on inflammation and coagulation in participants with impaired glucose tolerance. Diabetes 54:1566-1572

17. Selvin E, Paynter NP, Erlinger TP (2007) The effect of weight loss on C-reactive protein. Arch Intern Med 167:31-39

18. Fung TT, McCullough ML, Newby PK et al (2005) Diet-quality scores and plasma concentrations of markers of inflammation and endothelial dysfunction. Am J Clin Nutr 82:163-173

19. Schulze MB, Hoffmann K, Manson JE et al (2005) Dietary pattern, inflammation, and incidence of type 2 diabetes in women. Am J Clin Nutr 82:675-684

20. Kolb H, Mandrup-Poulsen T (2005) An immune origin of type 2 diabetes. Diabetologia 48:1038-1050

21. Van Gaal LF, Mertens IL, De Block CE (2006) Mechanisms linking obesity with cardiovascular disease. Nature 444:875-880

22. Krabbe KS, Pedersen M, Bruunsgaard H (2004) Inflammatory mediators in the elderly. Exp Gerontol 39:687-699

23. Licastro F, Candore G, Lio D et al (2005) Innate immunity and inflammation in ageing: a key for understanding age-related diseases. Immun Ageing 2:8

24. Ferrucci L, Harris TB, Guralnik JM et al (1999) Serum IL-6 level and the development of disability in older persons. J Am Geriatr Soc 47:639-646

25. Eriksson J, Lindström J, Valle T et al (1999) Prevention of type II diabetes in subjects with impaired glucose tolerance: the Diabetes Prevention Study (DPS) in Finland: study design and 1-year interim report on the feasibility of the lifestyle intervention programme. Diabetologia 42:793-801

26. Uusitupa M, Louheranta A, Lindström J et al (2000) The Finnish Diabetes Prevention Study. Br J Nutr 83(Suppl 1):S137-S142

27. Lindström J, Eriksson JG, Valle TT et al (2003) Prevention of diabetes mellitus in subjects with impaired glucose tolerance in the Finnish Diabetes Prevention Study: results from a randomized clinical trial. J Am Soc Nephrol 14:S108-S113

28. Laaksonen DE, Lindström J, Lakka TA et al (2005) Physical activity in the prevention of type 2 diabetes. The Finnish Diabetes Prevention Study. Diabetes 54:158-165
29. Lakka TA, Venäläinen JM, Rauramaa R, Salonen R, Tuomilehto J, Salonen JT (1994) Relation of leisure-time physical activity and cardiorespiratory fitness to the risk of acute myocardial infarction. N Engl J Med 330:1549-1554

30. Lindström J, Louheranta A, Mannelin M et al (2003) The Finnish Diabetes Prevention Study (DPS): lifestyle intervention and 3-year results on diet and physical activity. Diabetes Care 26:3230 3236

31. Ovaskainen ML, Valsta L, Lauronen J (1996) The compilation of food analysis values as a database for dietary studies: the Finnish experience. Food Chemistry 57:133-136

32. Nicklas BJ, You T, Pahor M (2005) Behavioural treatments for chronic systematic inflammation: effects of dietary weight loss and exercise training. CMAJ 172:1199-1209

33. Fogarty AW, Glancy C, Jones S, Lewis SA, McKeever TM, Britton JR (2008) A prospective study of weight change and systematic inflammation over 9 y. Am J Clin Nutr 87:30-35

34. Weinstein AR, Sesso HD, Lee IM et al (2004) Relationship of physical activity vs body mass index with type 2 diabetes in women. JAMA 292:1188-1194

35. Hu FB, Willett WC, Li T, Stampfer MJ, Colditz GA, Manson JE (2004) Adiposity as compared with physical activity in predicting mortality among women. N Engl J Med 351:2694-2703

36. Petersen AMW, Pedersen BK (2005) The anti-inflammatory effect of exercise. J Appl Physiol 98:1154-1162

37. Kristiansen OP, Mandrup-Poulsen T (2005) Interleukin-6 and diabetes: the good, the bad, or the indifferent. Diabetes 54(Suppl 2):S114-S124

38. Kempf K, Rose B, Herder C et al (2007) The metabolic syndrome sensitizes leukocytes for glucose-induced immune gene expression. J Mol Med 85:389-396

39. Koenig K, Khuseyinova N, Baumert J et al (2006) Increased concentrations of C-reactive protein and IL-6 but not IL-18 are independently associated with incident coronary events in middleaged men and women. Results from the MONICA/KORA Augsburg Case-Cohort Study, 1984-2002. Arterioscler Thromb Vasc Biol 26:2745-2751

40. Thorand B, Baumert J, Koenig W et al (2007) Sex differences in the prediction of type 2 diabetes by inflammatory markers: results from the MONICA/KORA Augsburg case-cohort study, 19842002. Diabetes Care 30:854-860

41. Ajani UA, Ford ES, Mokdad AH (2004) Dietary fiber and Creactive protein: findings from the National Health and Nutrition Examination Survey data. J Nutr 134:1181-1185

42. Qi L, Van Dam RB, Liu S, Franz M, Mantzoros C, Hu FB (2006) Whole-grain, bran, and cereal fiber intakes and markers of systemic inflammation in diabetic women. Diabetes Care 29:207-211

43. Ma Y, Griffith JA, Chasan-Taber L et al (2006) Association between dietary fiber and serum C-reactive protein. Am J Clin Nutr 83:760-766

44. King DE, Egan BM, Woolson RF, Mainous AG 3rd, Al-Solaiman Y, Jesri A (2007) Effect of high-fiber diet vs a fiber-supplemented diet on C-reactive protein level. Arch Intern Med 167:502-506

45. Nilsson AC, Östmann EM, Holst JJ, Björck IME (2008) Including indigestible carbohydrates in the evening meal of healthy subjects improves glucose tolerance, lowers inflammatory markers, and increases satiety after a subsequent standardized breakfast. J Nutr 138:732-739

46. Lindström J, Peltonen M, Eriksson JG et al (2006) High-fibre, low-fat diet predicts long-term weight loss and decreased type 2 diabetes risk: the Finnish Diabetes Prevention Study. Diabetologia 49:912-920

47. King DE (2005) Dietary fiber, inflammation, and cardiovascular disease. Mol Nutr Food Res 49:594-600

48. Heinrich PC, Castell JV, Andus T (1990) Interleukin-6 and the acute phase response. Biochem J 265:621-636 